\title{
Road Safety Evaluation Based on Direct Approach for Identification Problems and Significance of Countermeasures
}

\author{
Muhammad Asim Azim ${ }^{1, \text { a }}$,Shamim Akhtar, ${ }^{2, b}$, ZHAI-Fengwen ${ }^{3,}$ \\ cand WANG Yangping ${ }^{4, \mathrm{~d}}$ \\ ${ }^{1}$ School of Electronics and Information Engineering, Lanzhou Jiaotong \\ University, \\ Lanzhou 730070, China \\ a*gis.edu_lzjtu@yahoo.com
}

\begin{abstract}
Karachi is the capital city of Sindh province and considers as a metropolitan city (of Pakistan) consist of huge population, faces high numbers of road causalities and/or accidents. Uncertain safety situation and rapid development in number of vehicles, the ratio of the vehicle accidents is increasing greatly. For improvement safety culture, joint with exact problem identification and determination of possible countermeasuresare very helpful for efficient safety system. Direct approach based on the accountability of road traffic causalities and/or accidents. Presently, it is very necessary to know what is the significance of actual problem and how these problems can be properly described. In order to improve safety culture it is necessary to describe proper flow by reasonable statistic evaluation regarding problems and possible countermeasures. Additionally, in which each problem can be described in ranked according to significance and severity. This approach is called diagnostic approach, applied to evaluate safety problems and possible countermeasures in Karachi (city) of Pakistan.
\end{abstract}

Keywords: Direct approach, evaluation, identification of problems, and countermeasures.

\section{Introduction}

Throughout the world, cost regarding road causalities are related with trauma recordadmission and putting enormous burden on low-income and less assetscountries. Road safety issues and weak safety structure have significant cause involving in road causalities, everywhere in the world, and currently road accidents could become the biggest reason for deaths because annually 1. 2 million people generally dies\&fiftymilliongets injuries, just because of roadtraffic accidents [1,2].Concerning World Bank statistical record the road related victims will boost upfrom year 2000 to 2020 (WHO 2004).Pakistan is 
usually suffering 100billion of rupees yearly in preservation side, as a result of lack of road safety.Whereas Karachi is the capital cityof Sindh province and considers as a metropolitan city (of Pakistan) faces high numbers of road causalities, because of having huge population.It is estimated by SRDO (Social Research and Development Organization) in 2012 that Karachi is facing high number of causalitiescomparatively other major cities of Sindh province, as shows in figure.1.Right geometric design joint with exact problem identificationare very helpful for efficient safety system. Direct approach evaluation method based on the accountability of road traffic causalities and/or accidents. Direct evaluation procedure mainly focuses on crash-frequency, rate, severity and statistic models. In order to develop safety culture it is necessary to describe proper flow by reasonable statistic evaluation regarding problems and countermeasures [3-5,7-11].

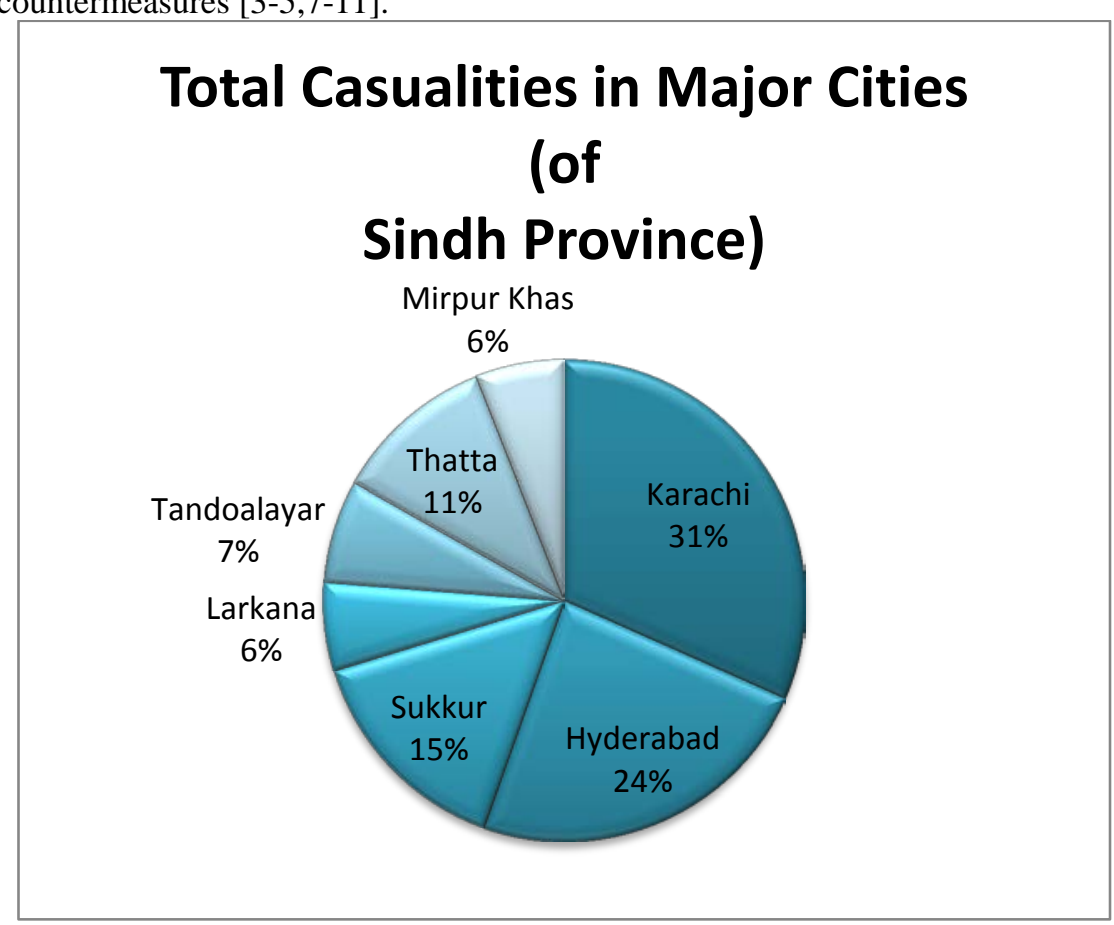

Figure 1: Road Casualities In Different Major Cities Of Sindh Province.

\section{Feature of Study Area}

Whole city spread over 3,527 sq. km. and fundamentally it is distributed into 18 towns, six cantonment boards and a few agencies. It is estimated by population census organization in 2011 that whole city has a projected population of 23.5 million and considered as 3rd largest city in the world by its population. As well as it is a vital city and positioned in the southern of region, with the Arabian 
Seashore. Karachi Geographic coordinates are $24^{\circ} 51^{\prime} \mathrm{N} 67^{\circ} 02 \mathrm{E}$, as well as de-merged into five original constituent districts, namely Karachi East, Karachi West, Karachi Central, Karachi South and Malir.

\section{Accident and/or casualty data}

There are several sources involving data, pertaining to instant vehicle accident registered information by police, trauma centers, insurance policies, and questionnaire as well as self-survey report information. All resources have their benefits and weakness. It must be stressed that these unusual databases are usually set way up for distinct purposes compared to traffic safety. For example; Police data itself is not done entirely for targeted safety requirements, just because of police workload issues. Many modest accidents can be handled by the conflicting parties. If the particular damage is small, then not even insurance organizations are informed as a result of no-claims bonuses. This specific results is a strongly to report more serious damage accident information, specially resulting in an opinion where certain accident information may be absent when compared with others. It is estimated that police are usually not professionals in accident accountability as well as medically and engineering expertise, so most of important information regarding accident are not be registered very accurate in documentation (Rosman, 1994). It is very clear that most of causalities and accident are reported by road traffic injury and prevention center in Karachi, Pakistan [6].All information regarding time, patient, causes, hospital and medical service is related to safety record. This safety record is very useful to find problems regarding safety improvements. As well as, very helpful to overview/ represent the local and/or national level actual safety situation [12,13], as shows in table and figure.2.

Table.1: Total Accident and Casualities-2013

\begin{tabular}{|c|c|c|c|c|c|}
\hline Month & Injuries & $\mathbf{\%}$ & Fatalities & $\mathbf{\%}$ & Total \\
\hline Fan & 2549 & $8 \%$ & 111 & $10 \%$ & 2660 \\
\hline Feb & 2629 & $8 \%$ & 86 & $8 \%$ & 2715 \\
\hline Mar & 2764 & $9 \%$ & 88 & $8 \%$ & 2852 \\
\hline Apr & 2279 & $7 \%$ & 49 & $4 \%$ & 2328 \\
\hline May & 2536 & $8 \%$ & 92 & $8 \%$ & 2628 \\
\hline Jun & 2744 & $9 \%$ & 100 & $9 \%$ & 2844 \\
\hline Jul & 2589 & $8 \%$ & 98 & $9 \%$ & 2687 \\
\hline Aug & 3341 & $10 \%$ & 122 & $11 \%$ & 3463 \\
\hline Sep & 3087 & $10 \%$ & 106 & $9 \%$ & 3193 \\
\hline Oct & 2901 & $9 \%$ & 104 & $9 \%$ & 3005 \\
\hline Nov & 2263 & $7 \%$ & 90 & $8 \%$ & 2353 \\
\hline Dec & 2498 & $8 \%$ & 84 & $7 \%$ & 2582 \\
\hline Total & 32180 & $100 \%$ & 1130 & $100 \%$ & 3310 \\
\hline
\end{tabular}




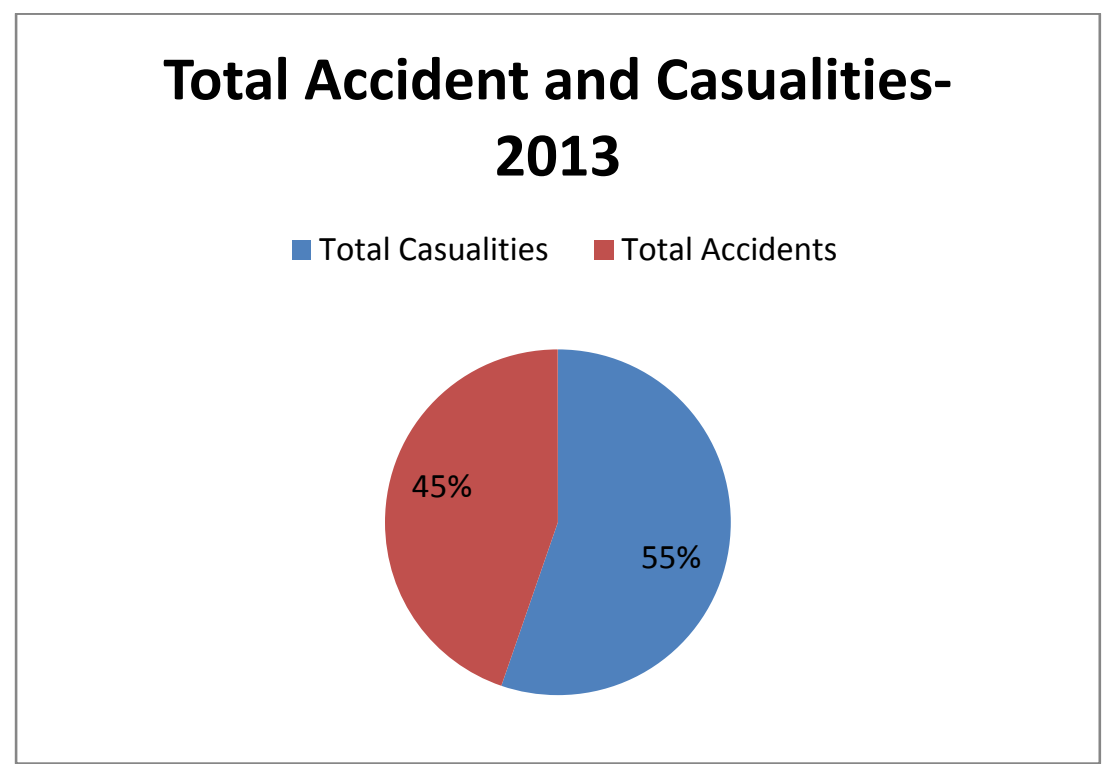

Fig.2: Total Accident and Casualities-2013

\section{Methodology}

With the rapid development in number of vehicles, the ratio of the vehicle accidents is increasing greatly.Presently, it is very necessary to know what is significance of actual problem and how these problems can be properly describe. Here, direct approach based on the accountability of road safety problems. For this purpose we must describe proper evaluation flow in which significant must be described regarding safety issues with significance. Additionally, each problem can be described in ranked according to significance and severity. Direct evaluation procedure mostly focuses on frequency, rate and severity. GenerallyIn order to development safety culture, it is necessary to describe proper flow by reasonable statistic evaluation. Safety problems with the uppermost ranks are identifying, possible countermeasures. Finally, possible countermeasures arerecommended safety description respectively major causes at high causalities and/or high accidents areas.

\section{Analysis}

For accountability, the data is confirmed by road traffic safety and prevention center Karachi.It is necessary to describe proper flow by reasonable statistic estimation based on direct approach for reasonable evaluation, in which significant must be describe regarding issues and countermeasures. In order to follow proper flow, first of all it is necessary to realize present situation. According to analysis result, 32,180 injuries and 1180 fatalities are reported in 
different trauma centers. Its means the situation is going worst day by day. There is no proper safety plan for accountabilityof these problems, so this research is very important regarding to observe present situation. Direct approach can be described in crash frequency, rate and severity, as shows in figure.3.

- Fatalities - Injuries

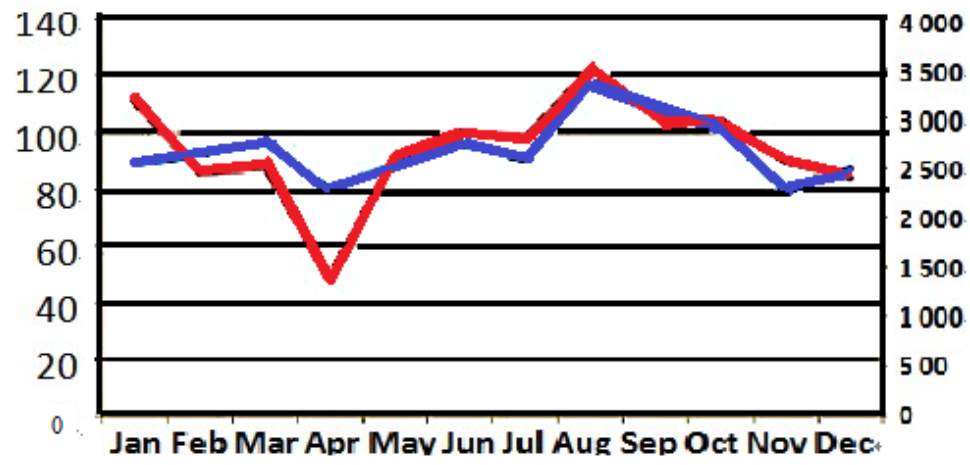

Fig.3: estimation of risk situation

After observation of safety situation,In order to compare and rank major safety problems, it's very important to find out the severity/rate/frequency of each contributory factor. Accident/causalities data is the basis for analysing and describing road traffic safety problems, as shows in fig. 4.

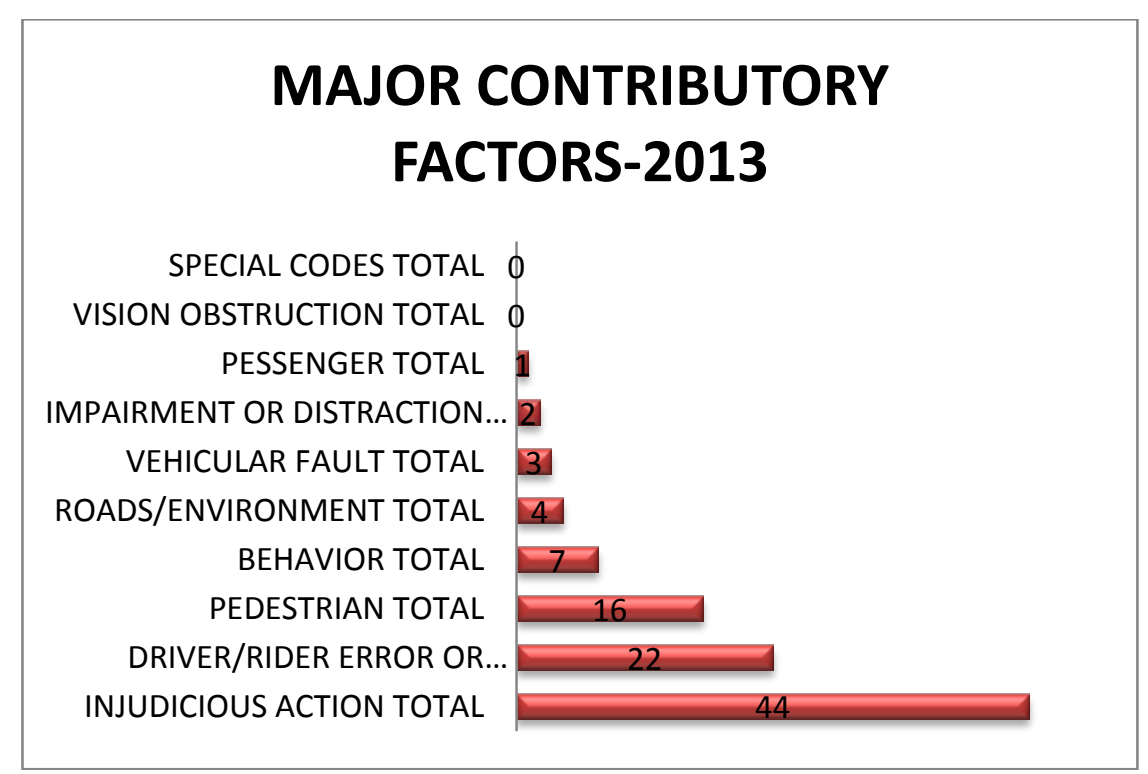

Figure.4: Roadsafety problems

According to this safety situation and identified safety problem, injudicious action is the most cause of casualties/fatalities with 44 percent by total no. of 
deaths/injuries. The determination of countermeasures must be formulated in prospective to control causalities/fatalities, and possible countermeasure can put forward to diagnostic issues by followingdirect approach evaluation process, as shows in figure.5.

Figure.5: Flow process of direct approachevaluation.

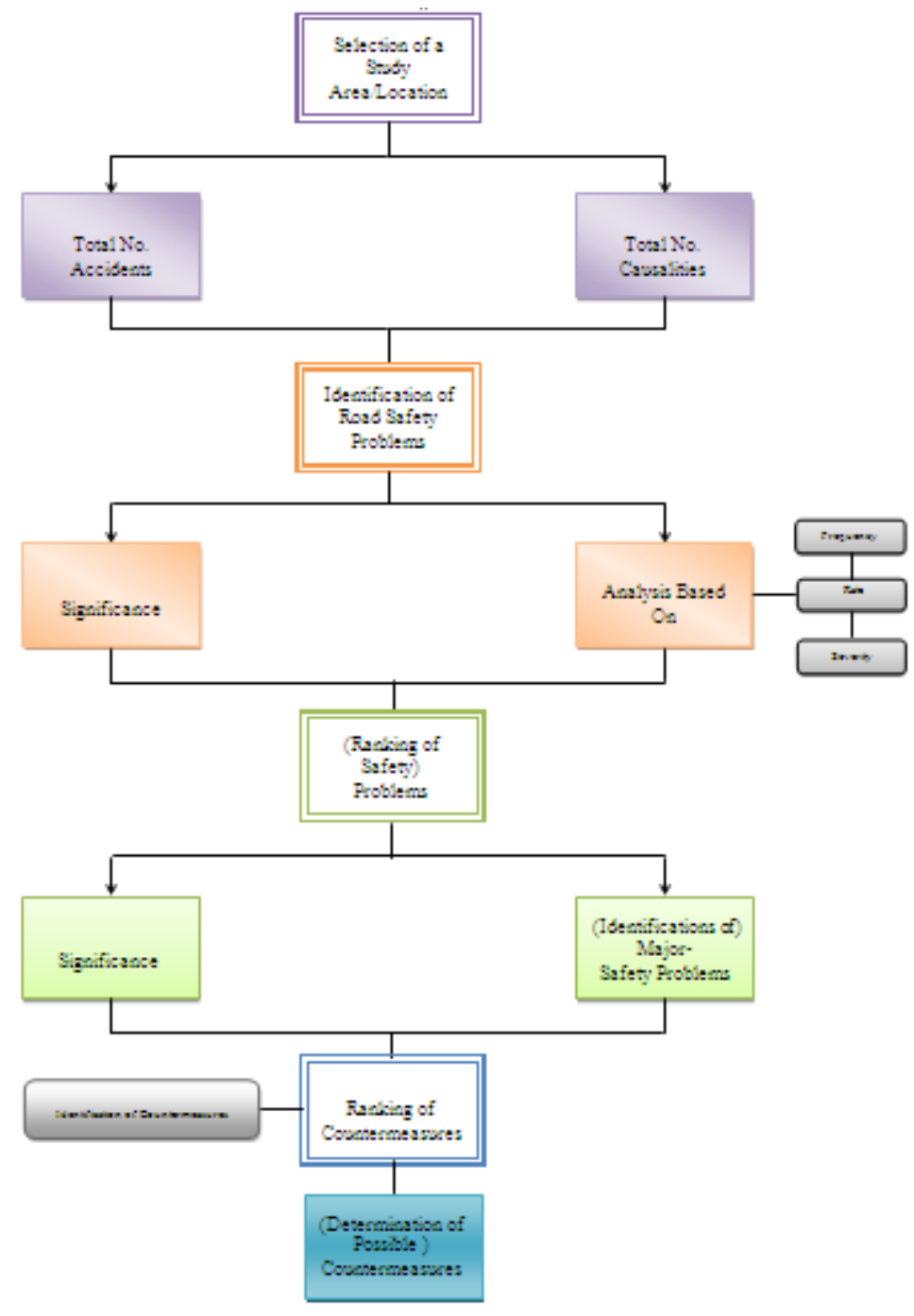




\section{Conclusion}

In order to improve safety culture it is necessary to describe proper flow by reasonable statistic evaluation. The main purpose of this study is to evaluatethe safety situation based on direct approach method. This approach is also known as diagnostic approach, can be better used in any study area safety for evaluation-situation, problems and description of possible countermeasures in flow.

\section{References}

[1] Razzak, J.A. and L. Laflamme, Limitations of secondary data sets for road traffic injuryepidemiology: a study from Karachi, Pakistan. Prehospital Emergency Care, 2005. 9(3): p.355-360.

[2] Ma Zengqiang, Pan Cunzhi, and, Wang Yongqiang, " Road Safety Evaluation from TrafficInformation Based on ANFIS ", Journal of TransportationEngineering, ASCE, 2008, pp. 554-558.

[3] Sheraz., E.B.A.L.A.E.K., Road Accident on Sindh,2012; Situation Analysis. Social Researchand Development Organization,SRDO., 2012. Traffic News \& Incident Reporting 2012 bySRDO: p. p.6.

[4] Shamim, S., et al., Initial results of Pakistan's first road traffic injury surveillance project.International journal of injury control and safety promotion, 2011. 18(3): p. 213-217.

[5] Yuan Li, Yuan Hewei, and Huang Zhengzhong,"Safety Improvement at Two-Way

StopControlledIntersection",JournalofTransportationEngineering,IEEE,2010,pp. 252-254.

[6] Ministry of Health Govt. of Sindh, P.S.B.A.F., ROAD TRAFFIC INJURY RESEARCH \&PREVENTION CENTRE, REPORT- 2013. Department of Neurosurgery, Jinnah Postgraduate Medical Centre, RafiquiShaheed Road, karachi-75510, Pakistan.

[7] Jian Lu, Li Yuan, Guoqiang Zhang, and Qiaojun Xiang, $i^{\circ}$ Diagnostic Approach for SafetyPerformance Evaluation and Improvements of Highway Intersection ", Journal of TransportationEngineering, TRB, 2007 Annual Meeting CD-Rom.

[8] Li Yuan, Jian Lu, Guoqing Zhang, and Qiaojun Xiang, " Safety Evaluation Approach ofHighway Intersection ", Journal of Transportation Engineering, ASCE, 2008, pp. 301- 307.

[9] Ma Zengqiang, Pan Cunzhi, and, Wang Yongqiang, " Road Safety Evaluation from TrafficInformation Based on ANFIS ", Journal of Transportation Engineering, ASCE, 2008, pp. 554-558.

[10] Zhu Shengxue, Lu Jian, Xiang Qiaojun, and, Yan Linli, " Rural Road Traffic SafetyDiagnosis Method " , Journal of Transportation Engineering, ASCE, 2009, pp. 4037-4042. 
[11] Sun, B.Y., Lu, J., and Ma Q.L., " Research on Method for Priorities of Traffic SafetyCountermeasures at Highway Intersections " , International Road SafetySeminar, Beijing, China,2005, pp. 341-344.

[12] Nieva, V. F., and J. Sorra. "Safety culture assessment: a tool for improving patient safety in healthcare organizations." Quality and Safety in Health Care12.suppl 2 (2003): ii17-ii23.

[13] Saunier, Nicolas, and TarekSayed. "Automated analysis of road safety with video data." Transportation Research Record: Journal of the Transportation Research Board 2019.1 (2007): 57-64. 